\title{
Comparison of Edge Detection Methods in Leaf Shape Analysis For Plant Classification
}

\author{
Manisha Amlekar ${ }^{a}$, Ashok Gaikwad ${ }^{a}$, Pravin Yannawar ${ }^{b}$, Ramesh Manza ${ }^{b}$ \\ ${ }^{a}$ Institute of Management Studies and Information Technology, Aurangabad, Maharashtra,India \\ ${ }^{b}$ Department of CSIT Dr. Babasaheb Ambedkar Marathwada University, Auranagabad, Maharashtra, \\ India
}

e-mail: manishaak2012@gmail.com,drashokgaikwad@gmail.com, pravinyannawar@gmail.com, manzaramesh@gmail.com

\begin{abstract}
In this research paper, comparison of edge detection methods is presented in leaf shape analysis for plant classification. Leaf is a very significant component of plant species that identify and classify the plants. Plant classification is the task performed by trained botanists and taxonomists. This task includes performing a set of operations. Because of this the task of classification of plants manually is time consuming. There are many biometric features of the leaves of the plants for classification. In this paper, Sobel and Canny edge detection methods are compared for their performance in leaf shape analysis operation for plant classification.
\end{abstract}

Keywords: plant classification, leaf shape, Sobel, Canny

\section{Introduction}

Plants are a very important part of our ecosystem. They maintain the balance in the environment. They are an important source of development of human being and living of them. Plants are useful for various industries. They are useful in medicines they are also useful as foodstuff. Plant classification is the process by which the plants' related group is collected, based on common characteristics, consisting of various tasks trained taxonomists and botanists follow. They follow many methods such as morphological method, cell and molecular biological approach. These methods are required to follow many critical tasks. Performing these tasks is a time consuming process and it requires many efforts. These processes are less efficient as it is difficult for one to remember all the species in the world. There are many features which classify the plant species, with the physiological characteristics of the leaf component being the most usuable features as they can be extracted easily. This paper presents the study of various techniques for shape features extraction for classification. These leaf shape features extraction eliminate the noise from the leaf image that reduces the leaf features which may make the classification complex and affect the efficiency of the classification model. The shape feature of leaf image is considered a type of crucial information that can be extracted by applying edge detectors with different methodology. This research paper presents a study of such edge detectors and their operation. Many researchers' previous works on shape features extraction are reviewed. Extraction of the shape of the leaf is nothing but segmentation 
of the leaf image, which determines and outlines the boundary pixels in order to extract the shape appropriately. This shape of the leaf is defined as the boundary. The edge is the boundary between two regions with relatively distinct gray level properties [5]. The shape extraction process examines the local discontinuity at each pixel element in an image. Orientation and location of a particular part of the leaf image is a part of interest for this examination 3. Based on these characteristics, the detector decides whether each of the examined pixels is part of the shape or not. Boundary pixels have the property of having gradual change in intensity and specific operators are required to determine such gradual change for defining the boundary pixel. Frei and Chen [3] presented morphological thinning and linking process to optimize the boundaries. The overview of first and second order derivative edge detectors and their performance evaluated in Vairalkar and Nimborkar [4] is presented and the color image segmentation using Sobel operator. Kaur and Malhotra [2] presented the use of canny for remote sensing application.

\section{Methodology}

The gradient based edge detection methods are studied for plant classification for analysis of shape of the leaf image. Gradient is nothing but the change in the intensity of the pixel in an image. Gradient based methods detect the shape by looking for the maximum and minimum in the first derivative of the image. These methods evaluate the gradient of the image generated along with two orthogonal directions. In these methods presence of the component for leaf shape as boundary pixels are predicted if the gradient of the image exceeds the predefined threshold value, $T$. The gradient can be computed by finding the derivatives along both orthogonal axes $x$ and $y$, where $f$ is the image, $\nabla f$ is change in intensity at $x$ and $y$ direction.

The gradient is estimated in a direction normal to the gradient of the boundary pixels. Then the magnitude of the gradient is computed and used for approximating boundary pixels as part of leaf shape. These techniques are processed on the leaves of the various species of the plants. These are the images of the leaves of the different species for finding their shape features.

$$
\nabla f=\left[\begin{array}{l}
G_{x} \\
G_{y}
\end{array}\right]=\left[\begin{array}{l}
\frac{\partial f}{\partial x} \\
\frac{\partial f}{\partial y}
\end{array}\right]
$$

Sobel edge detection - In this method, the edge detection is carried out by discrete differentiation operator. There is computation of the partial derivation in gradient that is approximated for finding the edge features of the leaf images. This technique performs $2 \mathrm{D}$ spatial gradient measurement on an image that corresponds to boundary pixels of the leaf images which might be further detected as leaf shape. Typically it is used to find the approximate absolute gradient magnitude at each point in an input grayscale image. This method finds the gradient measurement at each orientation of $G_{x}$ and $G_{y}[\underline{1},[3]$. These can be combined to find the absolute magnitude of the gradient at each point and the orientation of that gradient.

Canny edge detection - The Canny edge detection method finds edges by looking for the local maxima of the gradient of the input image. It calculates the gradient using the derivative of the Gaussian filter. The Canny method uses two thresholds to detect strong and weak edges. It includes the weak edges in the output only if they are connected to strong edges. As a result, the method is more robust to noise, and more likely to detect true weak edges. It smoothes the image by Guassian filter to remove the noise. Intensity gradient is then found for the leaf image. It applies non-maximum suppression to get rid of spurious response to detect edges for shape then applies double threshold to determine potential edges and then finalizes the boundary pixels for the shape component of the leaf image. 


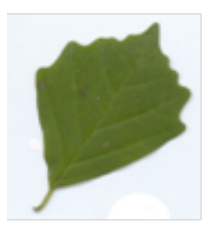

Original

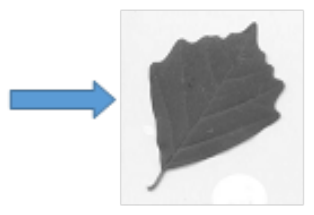

Grav level

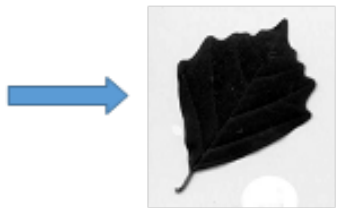

Enhanced
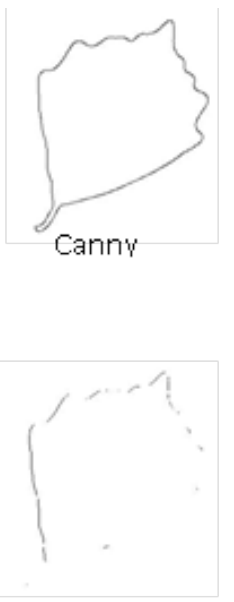

Sobel

Figure 1. Processing steps.

\section{Experimental Work}

In this experiment the images are taken from the ICL online database. This database provides various image samples of the leaves of the 221 different plant species. Database is published by Intelligent Computing Laboratory, Chinese Academy of Sciences 2010-2012. Figure 1 shows processing steps of the experiment which are carried out for the leaf shape analysis for plant classification. Initially, the leaf images are pre-processed so that they are all in the same format for further processing. As the color features of the leaves are varying, as per growth and the effect of sunlight, this pre-processing is required. In this step, leaf images are processed to get their gray level format then the resulting image is enhanced by increasing contrast. This resultant leaf image is further processed to get the leaf shape features with Canny and Sobel edge detection methods. Figure 1 shows the result of shape features by both methods. It is obvious that Canny method shows better performance for leaf shape extraction. This process is applied on 16848 leaf image samples. It properly identifies and detects the presence of boundary pixels for detecting the shape of the leaves. This method finds the boundary pixels by looking for local maxima of the gradient of the pre-processed leaf image $f(x, y)$. This method performs double thresholding for finding and detecting the strong and weak features of the leaf image and the features which show strong connectivity with the boundary pixels are supposed to detect the leaf shape pixel features. This implementation shows the Canny method resulted the best performance both visually and quantitatively. Table 1 shows quantitative experimental results of these methods. Table 1 shows that the performance of Canny method is better to analyse the leaf shape features for plant classification. In this table, the peak signal noise ratio, the maximum absolute error, the mean square error and the approximation ratio quantitative factors are shown. Statistical information of these factors shown that the Canny method results more peak signal noise ratio, less mean square error, less maximum absolute error and more approximation ratio than the respective findings for the Sobel method.

\section{Conclusion}

This experiment is performed to study the performance of the edge detection method Sobel and Canny for leaf shape analysis for plant classification. In this experiment the methods are evaluated by setting the threshold of 0.3. In Table 1 is shown that the Canny method has been evaluated with better result 
Table 1. Quantitative result for leaf shape analysis.

\begin{tabular}{|c|l|c|c|c|c|}
\hline Edge Detection Method & Quantitative Quality Measures & Minimum & Maximum & Average & Standard Deviation \\
\hline \hline \multirow{4}{*}{ Sobel } & Peak Signal Noise Ratio & 0.095 & 3.25 & 1.183 & \\
\cline { 2 - 6 } & Mean Square Error & 30700 & 63600 & 49816.613 & 5322.388 \\
\cline { 2 - 6 } & Maximum Absolute Error & 241 & 255 & 254.923 & 0.525 \\
\cline { 2 - 6 } & Approximation Ratio & 0 & 0.083 & 0.008 & 0.005 \\
\hline \multirow{4}{*}{ Canny } & Peak Signal Noise Ratio & 0.103 & 3.28 & 1.199 & 0.481 \\
\cline { 2 - 6 } & Mean Square Error & 239 & 255 & 254.919 & 0.0316 .173 \\
\cline { 2 - 6 } & Maximum Absolute Error & 0.004 & 0.104 & 0.016 & 0.544 \\
\cline { 2 - 6 } & Approximation Ratio & & 0.007 \\
\hline
\end{tabular}

than the Sobel method as it performs double thresholding for smoothing the boundary pixel for detecting them as part of the leaf shape of the plant species. This experiment is performed on leaf images of various species of the plants. The performance of the Canny algorithm relies mainly on the changing parameters of threshold values. The Canny edge detection method has a better performance.

In Table 1 visible results of performance of the edge detection methods are shown. The Sobel edge detection method results accurately with approximated edges for leaf shape about horizontal and vertical direction and the Canny edge detection approximates the edges by double thresholding to determine boundary pixels for detecting them as part of the leaf shape of the plant species. The performance of the Canny algorithm relies mainly on the changing parameters of threshold values. The Canny operator has a better performance in leaf shape analysis for plant classification.

\section{ACKNOWLEDGEMENT}

We specially acknowledge to Intelligent Computing Laboratory, Chinese Academy of Sciences as they provided the database for this research work. We acknowledge to Dr. Milind Desai.

\section{References}

[1] J. Hossain and M.A. Amin. Leaf shape identification based plant biometrics. In 13th International Conference on Computer and Information Technology (ICCIT), pages 458-463, 2010.

[2] K. Kaur and S. Malhotra. A survey on edge detection using different techniques. International Journal of Application or Innovation in Engineering and Management, 2(4):496-500, 2013.

[3] J. Pan and Y. He. Recognition of plant by leaves digital image and neural network. In International Conference on Computer Science and Software Engineering (CSSE), volume 4, pages 906-910, 2008.

[4] M.K. Vairalkar and S.U. Nimbhorkar. Edge detection of images using Sobel operator. International Journal of Emerging Technology and Advanced Engineering, 2(1):291-293, 2012.

[5] N. Valliammal and S.N. Geethalakshmi. Plant leaf segmentation using non linear K means clustering. International Journal of Computer Science Issues, 9(3):212-218, 2012. 